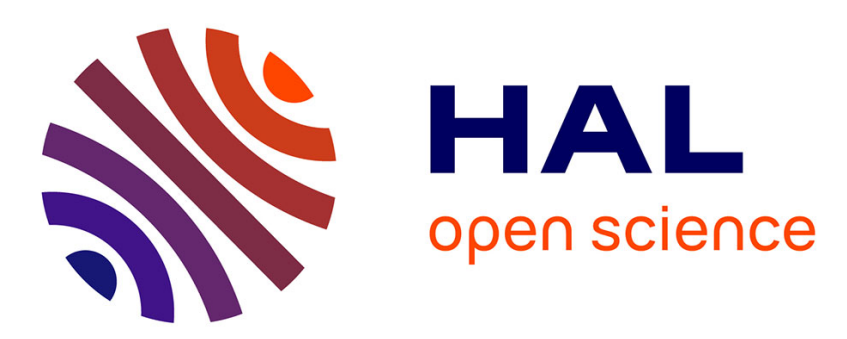

\title{
Influence of the molding pressure on the magnetic properties of soft ferrite composite materials
} Alexis Chevalier, F. Le Floc'H, Anne Marie Konn, Marcel Le Floc'H

\section{To cite this version:}

Alexis Chevalier, F. Le Floc'H, Anne Marie Konn, Marcel Le Floc'H. Influence of the molding pressure on the magnetic properties of soft ferrite composite materials. Journal de Physique IV Proceedings, 1998, vol.8 (Pr.2), pp.417-420. hal-00802175

\section{HAL Id: hal-00802175 \\ https://hal.univ-brest.fr/hal-00802175}

Submitted on 19 Mar 2013

HAL is a multi-disciplinary open access archive for the deposit and dissemination of scientific research documents, whether they are published or not. The documents may come from teaching and research institutions in France or abroad, or from public or private research centers.
L'archive ouverte pluridisciplinaire HAL, est destinée au dépôt et à la diffusion de documents scientifiques de niveau recherche, publiés ou non, émanant des établissements d'enseignement et de recherche français ou étrangers, des laboratoires publics ou privés. 


\title{
Influence of the molding pressure on the magnetic properties of soft ferrite composite materials
}

\author{
A. Chevalier, F. Le Floc'h*, A.M. Konn Martin and M. Le Floc'h \\ LEST, UMR 6616 du CNRS, 6 avenue Le Gorgeu, BP. 809, 29285 Brest cedex, France \\ * UMR 652, Chimie-Électrochimie Moléculaire-Chimie Analytique, 6 avenue Le Gorgeu, \\ BP. 809, 29285 Brest cedex, France
}

\begin{abstract}
Experimental studies on the complex susceptibility of soft magnetic composite materials ( $\mathrm{Ni}$ and $\mathrm{Ni}-\mathrm{Zn}$ spinels and YIG) in their region of spin resonance (SR) show that the SR frequency is a decreasing function of the magnetic concentration (demagnetising effects) which however tends to the natural spin resonance (NSR) frequency of the bulk material much before the unit concentration is reached. We take benefit of this remarkable property to show how powders could advantageously replace sintered materials to measure with no ambiguity the NSR frequencies. Since composite materials are achieved by molding processes under pressures, particular care of the technological stresses has been taken .
\end{abstract}

\section{INTRODUCTION}

Magnetic composite materials are manufactured by mixing both magnetic and non-magnetic powders in such proportions that it leads (at least for ceramic particles) to materials whose volume concentration can easily be changed from some fractions of percents to about $60 \%$. However, the possibility to reach fairly high magnetic loads of course depends on the magnitude of the molding pressure so that the magnetoelastic effects due to the stamping phase has to be regarded very carefully.

The heat treatment is surely both the more usual and the more effective route to cancel such technological stresses, but particular conditions concerning the binder can make this method quite inadequate for two reasons : (i) - An organic substance is used, but it can not withstand excessive temperature elevations. (ii) - A mineral is used but the heat treatment may involve sufficient dimensional variations to make samples unusable with regard to measurement techniques like coaxial line methods which are known to require precisely sized samples.

This paper deals with investigations on the Spin Resonance (SR) of different types of soft ferrimagnetic powders. They are different in their chemical natures (YIG, nickel and nickel-zinc spinels), as well as in their preparation modes (powders obtained either by grinding sintered ferrites or by coprecipitating methods).

Regarding ground powders, it is known that residual stresses are able to partially screen the intrinsic magnetic properties by introducing additional anisotropy terms, and this is particularly true in stresssensitive magnetic materials for which the NSR (Natural Spin Resonance) measured frequency becomes automatically wrong. This is a real problem when the goal is to have access to the intrinsic value of the effective anisotropy field $H_{K}$. Through a study of the stamping effects observed on the high frequency susceptibility in the SR region, this work shows how powders can become an elegant way to get rid of the problem of the uncontrolled internal stresses.

\section{EXPERIMENTAL RESULTS}

In our laboratory working on isotropic soft magnetic composite materials for several years, we currently use a phenomenological law (eq.1) for investigating the so-called "effective susceptibility » $\chi$ of magnetic mixtures as a function of their magnetic load $C$. This law has been established on two basic parameters : the first one, noted $\chi_{i}$ and called "intrinsic susceptibility», characterises the chemical 
nature of the magnetic substance. It was assigned to the rotational magnetisation [1]. The second parameter, noted $N$ and called the "effective shape factor ", is a global representation of the distributed demagnetising effects.

$$
(1-N) \chi^{2}+\left[1+(N-C) \chi_{i}\right] \chi-C \chi_{i}=0
$$

Eq.(1) is a useful way for predicting responses of magnetic powder materials to external parameters whose action on the intrinsic susceptibility $\chi_{i}$ is already known. Effects of temperature is an example which has been successfully treated previously $[2,3]$. The frequency influence, particularly in the SR region, is another example which has moreover been discussed in many other works [4].

Different models to describe the frequency-dependence of the rotational process of magnetisation exist. Since they lead in general to quite similar conclusions, the well known Gilbert-modified Landau and Lifshitz equation [5] has finally been retained for simulating the intrinsic frequency variations of our complex factor $\chi_{i}$. Investigating the responses obtained by solving eq.(1) has led us to point out a singular property of the SR frequency $F$ in relation with the magnetic fraction $C$ [6].

As the theoretical result of figure1-a shows, the SR frequency is normally increased by a decreasing of $C$, but it surprisingly tends towards a constant value equal to that of the bulk material, namely the NSR frequency, much before the unit concentration is reached. The threshold of concentration beyond which the SR frequency remains constant was noted $C_{p}$, and it was currently found about $0.3[6,7]$.

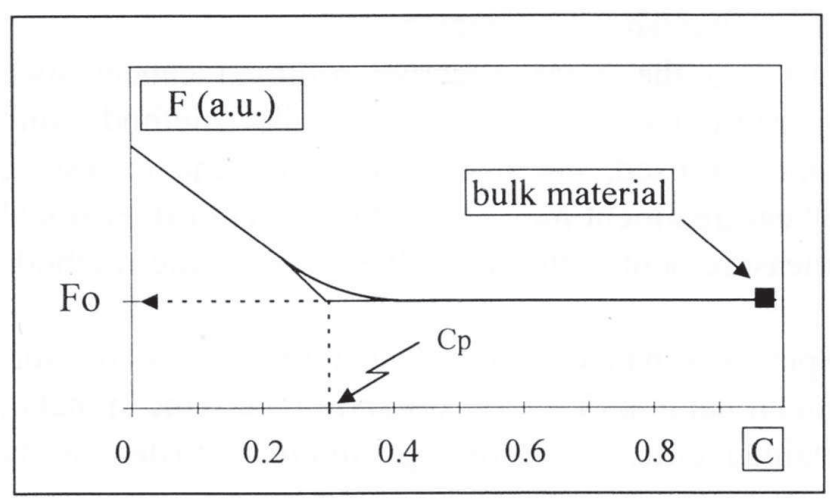

(a)

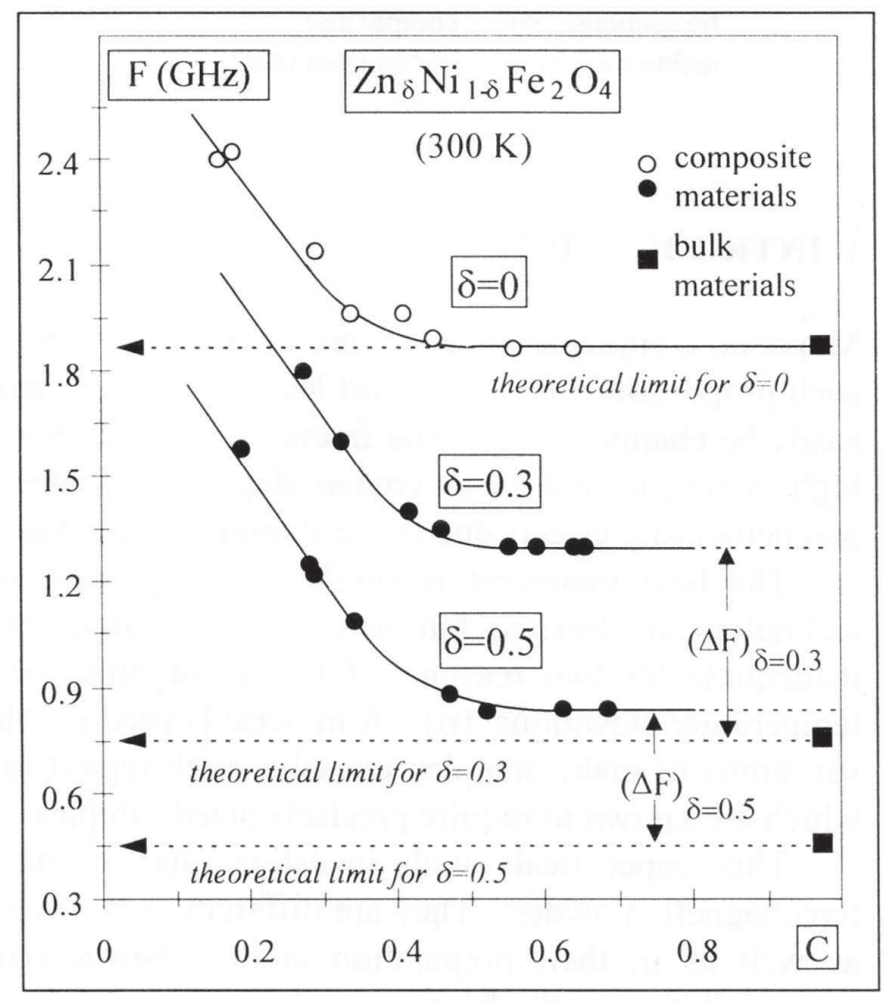

b)

Figure 1 - a) :Theoretical variations of the (SR) frequency in soft ferrimagnetic composite materials as a function of the magnetic load (result obtained by solving eq.1). - b) : Experimental confirmations of the theoretical result shown in (a) however with a difference appearing in the frequency values which will be explained further by the presence of technological stresses. The fact that this discrepancy does not exist in the nickel ferrite comes probably from the magnitude of its proper anisotropy field.

Fig. 1-b gives a typical experimental example of the SR frequency variations as a function of the concentration in spinel ferrites $\left(\mathrm{Zn}_{\delta} \mathrm{Ni}_{1-\delta} \mathrm{Fe}_{2} \mathrm{O}_{4}\right)$. In all cases, the threshold $\mathrm{C}_{p}$ is easily visible and clearly close to the above value 0.3 . These results moreover confirm that the NSR frequency (measured on sintered ferrites) is effectively reached for magnetic loads much smaller than the unit.

Notice that the above property is only achieved in the case of the nickel ferrite powder $(\delta=0)$. Concerning now nickel-zinc ferrite powders $(\delta=0.3, \delta=0.5)$, if the general aspect of curves is still preserved, the frequency limits are found higher (see the magnitudes of the discrepancies $(\Delta F)_{\delta}$ ) than the NSR frequencies. 
Residual technological stresses being probably the cause of the discrepancies observed, series of samples moulded under uniaxial pressures between 15 and $150 \mathrm{MPa}$ (pressure is applied on the resincoated particles only during the molding phase) have been prepared then investigated in the SR region. In all samples, magnetic loads were chosen sufficiently higher than the threshold $C_{p}$ to be sure that the SR frequencies could not be influenced by shifts of concentration due to the increasing molding.pressures. We take here a big advantage of the fact that, above $C_{p}$ the resonance frequency $F$ no longer depends on the magnetic load (see fig. 1), so that materials still remain comparable.

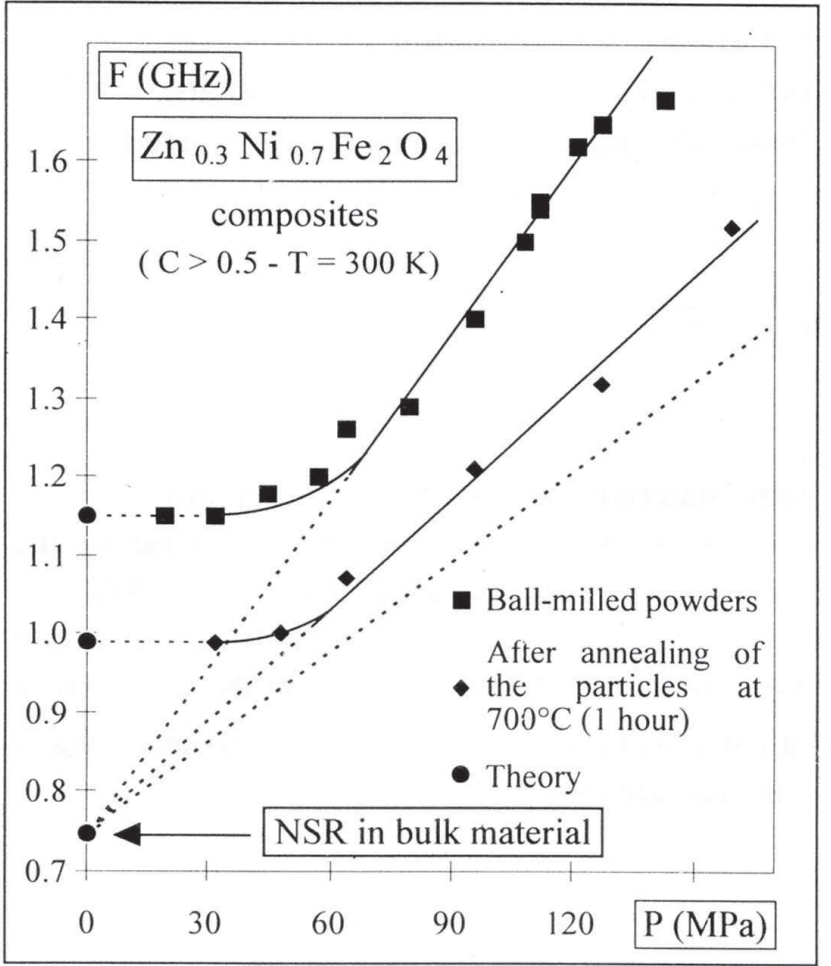

a)

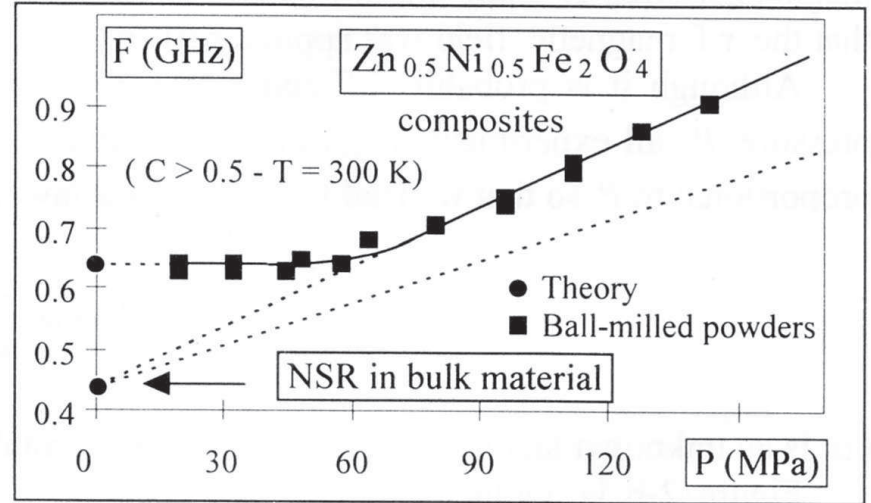

b)

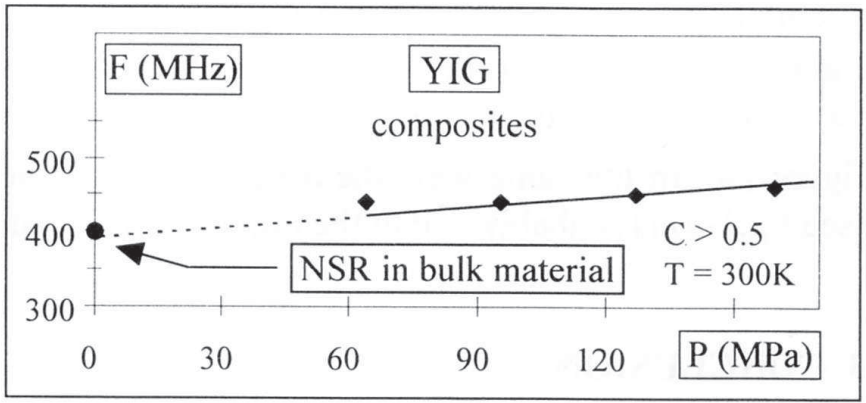

c)

Figure 2 - a): Effect of the molding pressure (only applied during the molding phase) on the SR frequency of soft ferrimagnetic ( $\mathrm{Ni}-\mathrm{Zn}: \delta=0.3$ ) composite materials. The SR frequency increases linearly with the compression strength only beyond a threshold $(50 \mathrm{MPa})$ corresponding to permanent internal stresses. This is well confirmed by annealing $\left(1 \mathrm{~h} . / 700{ }^{\circ} \mathrm{C}\right)$. Whatever the treatment applied on materials, the linear parts of curves converge towards the NSR frequency (that of the sintered ferrite). - b): Another example of Ni-Zn ferrite with an higher zinc content $(\delta=0.5)$. It is known that this modification decreases the effective anisotropy field and consequently the NSR frequency (450 Mhz [8]). Figure shows results which are consistent with that. - c): The third example deals with a well known ferrimagnetic garnet (YIG). Besides the previous remarks, the tenuous effects observed in this last case are simply explainable by the small value of the ratio $\lambda_{S} / M_{S}$.

Through the results obtained on $\mathrm{Ni}-\mathrm{Zn}(\delta=0.3)$ composite materials, figure 2-a shows how the pressure only applied during the molding phase, may have a persistent action on the value of the SR frequency. Two regions appear in which the behaviours are very different: Below $50 \mathrm{MPa}$, the SR frequency does not depend of the compression strength, whereas beyond, it increases linearly. On the same figure, near the results concerning ball-milled powders, those of annealed particles $\left(700^{\circ} \mathrm{C} / 1\right.$ hour $)$ have been reported leading to the expected conclusion that annealing reduces the amount of internal stresses and simultaneously reduces the SR frequency (the change in slopes is not yet explained). Anyway, whatever the treatment applied to the particles during the manufacturing process, the straight lines converge towards the value of the NSR frequency of the bulk material (750 MHz for $\delta=0.3$ [8]). As a comparison, the doted line is the theoretical response (see eq.1) of an hypothetical material in which 
particles would be submitted to internal stresses $\sigma_{i}$ all applied in the magnetisation direction (say along one of the easy axes). The straight line naturally starts from the NSR frequency $F_{0}$ of bulk:

$$
F=F_{0}+\frac{3 \gamma}{\mu_{0}}\left(\frac{\lambda_{S}}{M_{S}}\right) \sigma_{i}
$$

$\gamma$ is the gyromagnetic ratio, $\mu_{0}$ is the vacuum permeability, $\lambda_{S}$ and $M_{S}$ are respectively the saturation magnetostriction constant and the saturation magnetisation. Eq.(1) has been obtained simply by assuming that the r.f. magnetic field was applied perpendicularly to the static magnetisation.

Although it is probably difficult to theoretically correlate the internal stresses $\sigma_{i}$ to the molding pressure $P$, all experiments (fig.2) allow to think that, inside the domain of strength investigated, $\sigma_{i}$ is proportional to $P$ so that we lead to an empirical law fairly similar to eq.(1):

$$
F=F_{0}+\alpha \frac{3 \gamma}{\mu_{0}}\left(\frac{\lambda_{S}}{M_{S}}\right) P
$$

( $\alpha$ is an unknown factor to be determine experimentally).

Figure 2-b is another example still concerning spinel materials, but with an higher zinc content $(\delta=0.5)$. It is known that such a change in the chemical composition involves the decrease of the effective anisotropy field [9] and the simultaneous decrease of the NSR frequency (450 Mhz [8]). The figure gives a good confirmation of that. Yttrium-iron garnets (YIG) have intrinsic constants giving a ratio $\left(\lambda_{S} / M_{S}\right)$ much smaller than in Ni-Zn spinels. This is consistent with the very weak slope observed in figure 2-c. In the same way, the absence of magnetoelastical effects observed on nickel ferrite powders (see fig.1-b) is probably due to the big initial value of its intrinsic anisotropy.

\section{CONCLUSION}

Magnetic powders have SR frequencies which tend towards the NSR frequency of bulks (sintered materials) for concentrations just beyond about 0.3. Powders seem to be thus a very interesting aspect of matter to develop useful methods for investigating effective anisotropy fields by high frequency measurements. Concerning the difficult question of stresses induced by the molding phase, it has been shown how a simple technique of extrapolation can lead to the expected NSR frequency.

\section{References}

[1] Le Fur P., Konn A.M., Laurent P., Le Floc'h M., Le Floc'h F., Binon P., J.de Phys.IV France 7 (1997) C1-545.

[2] Le Floc'h M., Konn A.M., Talbot Ph., Mattei J.L., Menexiadis G., Le Floc'h C., C. Bayle, J. Of M.M.M. 104-107 (1992) 1591-1592.

[3] Laurent P., Thesis, Université de Bretagne Occidentale (Brest) 1994.

[4] Berthault A., Rousselle D., G. Zerah, J. Of M.M.M. 112 (1992) 477.

[5] Gilbert T.L., Phys. Rev., 100 (1955) 1243.

[6] Laurent P., Viau G., Konn A.M., Gelin Ph., Le Floc'h M. J. Of M.M.M. 160 (1996) 63-64.

[7] Chevalier A., Le Floc'h F., Mattei J.L., Talbot Ph., Le Floc'h M., « Spin resonance in soft magnetic composite materials : a surprising effect of the magnetic load», SMM13-Grenoble 24-26 September 1997.

[8] Gieraltowski J., J. de Phys. IV France, 38 (1977) C1-57.

[9] Globus A., Duplex P., J.Of Appl. Phys., 39 (1968) 727-729. 